\title{
CONHECIMENTO SOBRE TRIAGEM NEONATAL E SUA OPERACIONALIZAÇÃO*
}

Ivanete da Silva Santiago Strefling ${ }^{1}$, Xênia Martins Monfrim² ${ }^{2}$, Wilson Danilo Lunardi Filho ${ }^{3}$, Karen Knopp de Carvalho ${ }^{4}$, Ana Lucia Soares de Azevedo ${ }^{5}$

\begin{abstract}
RESUMO: Estudo exploratório-descritivo, de abordagem qualitativa, que objetivou descrever o conhecimento das enfermeiras sobre a triagem neonatal e sua operacionalização. Participaram do estudo 13 enfermeiras, atuantes em sete unidades de Estratégia de Saúde da Família em um município do sul do Rio Grande do Sul (RS), no período compreendido entre maio e julho de 2010. Os dados foram coletados em entrevistas semiestruturadas e analisados através da técnica do "Discurso do Sujeito Coletivo". As enfermeiras entrevistadas demonstraram conhecimento acerca do exame de Triagem Neonatal, especificamente quanto à sua finalidade, o período ideal para coleta de sangue e a técnica para coleta, assim como quanto às doenças que são rastreadas e quais delas o RS está habilitado para diagnosticar. No decorrer deste estudo, ressaltou-se a importância de o profissional da enfermagem buscar continuamente capacitação técnico-científica. Por outro lado, percebeu-se a necessidade dos gestores públicos proporcionem mais oportunidades de qualificação aos profissionais da saúde. DESCRITORES: Enfermagem; Triagem neonatal; Conhecimentos, atitudes e práticas em saúde.
\end{abstract}

\section{CONOCIMIENTO SOBRE EL TRIAR NEONATAL Y SU OPERACIONALIZACIÓN}

RESUMEN: Estudio exploratorio descriptivo, de abordaje cualitativo, que tuvo el objetivo de describir el conocimiento de las enfermeras sobre el triar neonatal y su operacionalización. Participaron del estudio 13 enfermeras actuantes en siete unidades de Estrategia de Salud de la Familia en un municipio del sur de Rio Grande do Sul, en el periodo de mayo a julio de 2010. Los datos fueron obtenidos en entrevistas semiestructuradas y analizados por medio de la técnica del Discurso del Sujeto Colectivo. Las entrevistadas demostraron conocimiento acerca del examen de triar neonatal cuanto a la finalidad, periodo ideal para recoger sangre, técnica para recoger, enfermedades rastreadas y cuales de ellas el Estado está habilitado a diagnosticar. A lo largo del estudio se destacó la importancia del profesional de la enfermería en buscar de modo continuo la capacitación técnico científica. Por otro lado, se percibió la necesidad de los gestores públicos de proporcionar más oportunidades de cualificación a los profesionales de la salud. DESCRIPTORES: Enfermería; Triar neonatal; Conocimientos, actitudes y prácticas en salud.

\section{KNOWLEDGE OF NEONATAL SCREENING AND ITS OPERATIONALIZATION*}

\begin{abstract}
This exploratory-descriptive study, with a qualitative approach, aimed to describe nurses' knowledge relating to neonatal screening and its operationalization. The study participants were 13 nurses working in seven Family Health Strategy Centers in a city in the south of the State of Rio Grande do Sul, in May - July 2010. The data were collected in semi-structured interviews and were analysed using the technique of the Discourse of the Collective Subject. The interviewees showed knowledge concerning the neonatal screening test regarding its aim, the ideal period for blood collection, the collection technique, the diseases tracked, and which of them the State is equipped to diagnose. During the study, the importance was emphasized of the nursing professional continuously seeking technical-scientific training. Concomitantly, the need was perceived for public managers to provide more training opportunities to the health professionals.
\end{abstract}

DESCRIPTORS: Nursing; Neonatal screening; Knowledge, attitudes and practices in health.

\footnotetext{
*Artigo original resultante da pesquisa intitulada "O Programa Nacional de Triagem Neonatal apresentada na disciplina de Políticas Públicas de Saúde - Mestrado em Enfermagem do Programa de Pós-Graduação da Universidade Federal do Rio Grande em 2012

${ }^{1}$ Enfermeira. Mestre em Enfermagem. Professora da Universidade da Região da Campanha. Pelotas-RS-Brasil

${ }^{2}$ Enfermeira. Hospital Universitário São Francisco de Paula. Mestre em Saúde e Comportamento. Pelotas-RS-Brasil

${ }^{3}$ Enfermeiro. Doutor em Enfermagem. Professor da Universidade Federal do Rio Grande. Rio Grande-RS-Brasil

${ }^{4}$ Enfermeira. Mestre em Enfermagem. Professora da Universidade Católica de Pelotas. Pelotas-RS-Brasil

${ }^{5}$ Enfermeira. Mestranda em Saúde e Comportamento. Professora da Universidade Católica de Pelotas. Pelotas-RSBrasil
} 


\section{INTRODUÇÃO}

O exame de Triagem Neonatal (TN), popularmente conhecido como teste do pezinho, é realizado mediante coleta de amostra de sangue do calcanhar do recém-nascido ${ }^{(1)}$. Trata-se de um exame laboratorial simples que detecta doenças metabólicas, genéticas e/ou infecciosas, antes que os sintomas tornem-se evidentes, facilitando a inclusão do recém nascido em tratamento específico a fim de diminuir ou eliminar sequelas associadas a cada doença, permitindo uma melhor qualidade de vida à criança ${ }^{(2)}$.

Desde a década de 1960, a Organização Mundial da Saúde vem preconizando programas populacionais de TN para a prevenção de deficiências mentais e agravos à saúde do recém-nascido. No Brasil, tal exame começou a ser utilizado na década de 1970 , sendo considerada uma iniciativa pioneira na América Latina ${ }^{(3)}$.

Na década de 1990 a TN foi incorporada ao Sistema Único de Saúde (SUS) e se expandiu para todo o território nacional, rastreando doenças como a fenilcetonúria e o hipotireoidismo congênito, incluídos pela Portaria GM/MS n $n^{\mathrm{o}} 22 / 92^{(2)}$. Em 2001, o Ministério da Saúde, lançou o Programa Nacional de Triagem Neonatal (PNTN) por meio da Portaria GM/MS n ${ }^{\circ}$ 822/01 que, além de acrescentar o rastreamento da anemia falciforme e a fibrose cística, objetivou ampliar a cobertura para $100 \%$ dos nascidos vivos, incluindo a realização do exame laboratorial, a busca ativa dos casos suspeitos, a confirmação diagnóstica e o tratamento e acompanhamento multidisciplinar especializado dos pacientes portadores de alguma das doenças triadas ${ }^{(1)}$.

Frente o processo de descentralização da gestão do sistema de saúde, em curso no Brasil, e com o propósito de atingir a pluralidade dos contextos vivenciados, o PNTN envolveu as estruturas públicas nos três níveis de governo, municipal, estadual e federal proporcionando uma mobilização ampla em torno das ações relacionadas à $\mathrm{TN}^{(1,3)}$.

Assim, ficou estabelecido que o PNTN seria executado de forma articulada pelo Ministério da Saúde e pelas Secretarias de Saúde dos estados e Distrito Federal através da criação de uma Rede Estadual de Triagem Neonatal, tendo como unidade central os Serviços de Referência em Triagem Neonatal (SRTN) ${ }^{(1)}$. Frente aos diferentes níveis de organização das redes assistenciais nos estados, da variação percentual de cobertura da TN que vinha sendo realizada em nascidos vivos e ainda da diversidade das características populacionais existentes no país, optou-se por implantar o PNTN em fases ${ }^{(3)}$.
A Fase I detecta a fenilcetonúria e o hipotireoidismo congênito. A fase II inclui as patologias da fase I e mais a anemia falciforme e outras hemoglobinopatias. A fase III passa a detectar as doenças rastreadas na fase I e fase II acrescentando o diagnóstico de fibrose cística $^{(2)}$. O credenciamento de cada fase depende da cobertura populacional de no mínimo $70 \%$ e da organização do SRTN. O Rio Grande do Sul está credenciado à fase II do PNTN ${ }^{(3)}$.

No que se refere à captação da amostra de sangue, esta deve ser realizada a partir de 48 horas após a ingestão proteica pelo recém-nascido. Desde então, deve ser feita a coleta o mais rápido possível, pois, como as doenças rastreadas são assintomáticas, quanto antes forem diagnosticadas, mais precocemente poderão ser iniciados os respectivos tratamentos, prevenindo agravos irreversíveis ao desenvolvimento físico e mental da criança $a^{(1-4)}$.

Diante do exposto, acredita-se que ações preventivas configuram uma das melhores estratégias para evitar a morbimortalidade infantil ${ }^{(5)}$. O exame de TN é uma medida preventiva que compete à enfermeira, que tem fundamental participação no processo de implementação do PNTN, esclarecer aos cuidadores sobre a importância e a finalidade do exame, do procedimento em si, bem como, da necessidade de buscar o resultado ${ }^{(5-6)}$.

Deste modo, acredita-se que o momento da coleta é ocasião oportuna para a enfermeira interagir com os pais e reforçar orientações que "permitam à família a sensação de segurança, oferecendo a ela saberes que consolidam a responsabilidade no sentido de promover o bem-estar e apoio à saúde de seu filho"(6:600). Além da necessidade de orientação à família, a enfermeira também tem papel importante enquanto multiplicadora de conhecimento na equipe de saúde com a qual trabalha ${ }^{(5)}$.

Todavia, apesar da relevância do assunto, há escassez de produções científicas que tratem da atuação da enfermeira no programa de $\mathrm{TN}^{(3)}$. Em uma pesquisa realizada junto ao SRTN no Estado do Paraná, foi identificado alto índice de erros técnicos nas coletas e necessidade de repetição, por imperícia ${ }^{(6)}$. E mais, por meio de uma revisão integrativa realizada, verificou-se que diversos estudos trataram acerca do conhecimento dos pais a respeito da $\mathrm{TN}^{(5-9)}$, sendo possível averiguar que os pais detêm informações superficiais sobre a importância e finalidade do exame ${ }^{(7)}$.

Tais lacunas podem estar relacionadas à falta de conhecimento técnico-científico e/ou desvalorização do exame pelos profissionais que fazem a coleta ${ }^{(2,4)}$, 
o que denota a importância das enfermeiras estarem capacitadas, tanto para orientar os cuidadores, quanto para instruir o responsável pela coleta (amostra de sangue) de forma satisfatória ${ }^{(9)}$. A coleta realizada de maneira satisfatória e dentro do tempo hábil configurase como uma das etapas importantes para o sucesso dos objetivos propostos pelo PNTN ${ }^{(3-4)}$.

Deste modo, ao serem apresentadas evidências quanto à importância da enfermeira como agente propagador de informações e orientações nos diversos momentos de contato com os pais e junto à equipe de saúde, restou justificada a realização deste artigo que tem como objetivo descrever o conhecimento das enfermeiras sobre a triagem neonatal bem como sua operacionalização.

\section{METODOLOGIA}

Trata-se de um estudo exploratório-descritivo, de abordagem qualitativa, realizado durante os meses de maio e julho de 2010. Os sujeitos deste estudo foram 13 enfermeiras atuantes em sete das oito equipes de Estratégia de Saúde da Família (ESF) existentes na zona urbana de um município do Sul do Rio Grande do Sul, que gerenciam e/ou coletam amostras de sangue para o diagnóstico das doenças rastreadas pelo PNTN.

Os critérios adotados para a inclusão neste estudo foram: ser enfermeira/o; ser o procedimento desenvolvido pela equipe de ESF e ter disponibilidade e interesse em participar do estudo. Após esclarecimentos sobre o objetivo da pesquisa e assinatura do termo de consentimento livre e esclarecido, foram realizadas entrevistas semiestruturadas em local previamente combinado entre o pesquisador e a entrevistada.

O questionário para coleta de dados foi composto pelas seguintes questões: Para você o que é Triagem Neonatal? Como é a técnica utilizada na coleta de sangue para o teste do pezinho? Quais são as doenças detectadas na Triagem Neonatal? As entrevistas foram gravadas e depois transcritas para manter a fidedignidade das informações.

As informações foram interpretadas com base no método do Discurso do Sujeito Coletivo (DSC) ${ }^{(10)}$, que possibilitou construir, com recortes dos diversos discursos individuais, discursos-síntese que expressaram um pensamento coletivo das enfermeiras participantes do estudo, acerca do problema investigado.

Os dados foram apresentados em agrupamentos de ideias centrais (IC). Foram respeitados os preceitos da Resolução n ${ }^{\circ}$ 466/12, do Conselho Nacional de Saúde e obtida autorização da Secretária Municipal de Saúde, além de aprovação do Comitê de Ética em Pesquisa da Saúde, de acordo com o Parecer no 53/2009.

\section{RESULTADOS}

Dentre as enfermeiras atuantes nas unidades de ESF e que foram entrevistadas, todas mencionaram ter realizado pós-graduação em alguma área da saúde, não necessariamente em saúde coletiva ou saúde da família. Além disso, todas trabalhavam como enfermeira há cerca de dez anos. Entretanto, quanto à área de atuação, algumas referiram ter atuado, anteriormente, em instituições hospitalares. No que se refere à faixa etária, esta variou de 42 a 47 anos.

No que tange ao entendimento das entrevistadas em relação ao que se trata o exame de $\mathrm{TN}$, apesar da importância da enfermeira em possuir conhecimento sobre o assunto, para realizar o procedimento de forma eficaz e poder orientar adequadamente, percebeu-se a necessidade de maior aprofundamento das entrevistadas, conforme os discursos descritos na IC: finalidade da triagem neonatal segundo as enfermeiras:

Triagem neonatal são aqueles exames que são feitos logo que o bebê nasce, quando a criança chega aqui na UBS, também pode se realizar em hospital, para analisar o sangue do recém-nascido, rastrear, ver se tem algum problema, alguma má formação. Pode-se diagnosticar um problema mental que pode ser tratado e evitar um déficit cognitivo. Imagina poder tratar e a pessoa ficar normal. Também para saber quais doenças que o SUS contemple, e detectar algum dos traços das possíveis doenças metabólicas, congênitas que podem ser descobertas cedo, a tempo. Elas podem ser tratadas sem maiores danos para a criança no futuro. (E2, E3, E4, E7, E10, E11)

Sobre o período hábil para a realização da coleta da amostra de sangue, as enfermeiras mencionaram que a captação deve ser feita nos primeiros dias de vida do neonato, porém demonstraram algumas fragilidades quanto à orientação do período apropriado para tal coleta. Assim temos na IC: "Período ideal para coleta da amostra de sangue para o exame Triagem neonatal":

\section{$T N$ é feito do $3^{\circ}$ ao $7^{\circ}$ dia. Se faz no $R N$ de no máximo} dez dias. Depois do décimo se faz uma justificativa, se a criança ficou internada, ou é prematura uma coisa desse gênero, desde então tem que fazer o exame até 
30 dias. Hoje a gente não tem mais aquela urgência de coletar nos primeiros sete dias de vida que já existem estudos que dizem que o teste coletado nos 30 dias não traz prejuizo nenhum para a criança. Até um mês de idade a melhor idade de coletar diria que a partir de 4 dias, antes de 4 dias não da para a gente coletar, só em casos esporádicos se a criança teve internada e não deu para fazer, tem essas exceções, porque não da para a gente identificar o tipo de patologia que a criança irá ter. (E1, E3, E4, E5, E13)

Das enfermeiras entrevistadas, todas mencionaram já ter tido contato com orientações sobre a técnica correta para realizar a coleta da amostra sanguínea. Quando questionadas, além de apontar os passos realizados na coleta, observou-se ênfase quanto a interação com o familiar durante o procedimento:

A gente segue o protocolo. Nós já recebemos vários informes sobre a técnica do teste do pezinho, de como que se faz a coleta. Eu particularmente preparo o cartãozinho, preencho todos os dados de identificação da criança e da mãe. Explico para a mãe como vai acontecer, peço para a mãe, o pai ou o cuidador, colocar o nenezinho em pé no colo, que é melhor peito com peito, faço uma massagenzinha no pé, principalmente se for inverno, porque é mais dificil encher de sangue. Dai é feita então a coleta de sangue do pezinho. Calço a luva, faço assepsia com algodão seco, ai faço o " $V$ " no calcanhar esquerdo para não furar no meio, pois conforme nos passaram no curso que a gente fez na secretaria municipal de saúde, tem um local correto para se coletar. Com uma lanceta descartável ou uma agulha de HGT faço um piquezinho suficientemente bom, dou um leve giro, pois a ponta desta lanceta é meio triangular. Às vezes, quando há necessidade eu faço um segundo piquezinho no mesmo buraquinho porque a gente sabe que não pode esfregar para que a gota de sangue venha razoável, nem de menos $e$ nem fazer aquela borrada toda de sangue, são gotas médias. Às vezes, acontece do pé estar gelado e eu ter que ir para o outro pé para poder terminar com a técnica. Em seguida, ordenho o pezinho do bebê como se ordenha uma teta de vaca, vou ordenhando para que as gotinhas caiam sem encostar no papel, desprezo a primeira gota de sangue e tento preencher todos os campos necessários para a amostra, geralmente são de três a cinco espacinhos no papel filtro disponível na unidade. Terminado a coleta, boto um algodãozinho, fico pressionando um pouquinho e deito a criança com o pé para cima para impedir o sangramento, coloco a meia e explico para a mãe não ficar esfregando. Após colher as amostrinhas, estas são encaminhadas, através da secretaria da saúde, ao laboratório do estado e trinta dias após, vem o resultado para ver se a criança precisa fazer a recoleta ou o resultado esta normal. (E4, E8, E9, E11, E12, E13)

Ao ser questionado sobre as doenças cobertas pelo PNTN, a maioria das entrevistadas apontou três das patologias rastreadas e contempladas pela fase II, à qual o estado do Rio Grande do Sul está credenciado a diagnosticar e tratar. Tal ficou evidenciado na IC: Identificação das doenças rastreadas pelo exame de Triagem Neonatal:

Na rede detectamos três, mas a triagem neonatal engloba vários exames, dependendo do laboratório. $O$ nosso é o básico então ele ainda só cobre essas três doenças: Fenilcetonúria, hipotireoidismo congênito e anemia falciforme. (E5, E11, E12)

Em cada discurso, as entrevistadas expressaram também seu entendimento sobre cada uma das doenças triadas. Assim a IC: $O$ enfoque de cada doença rastreada pela $T N$ :

Que eu lembro, a fenilcetonúria vai rastrear se a criança tem possibilidade de ser intolerante a algum tipo de proteína é uma doença relacionada ao sistema nervoso central, detecta retardo mental; já o hipotireoidismo congênito é referente a estatura e ao peso dessa criança ela não vai ter um crescimento normal. Neste caso, o exame vai rastrear se os niveis hormonais da criança, produzidos pela glândula tireoide, estão adequados para o crescimento e desenvolvimento normal da criança. Também temos a anemia falciforme, doença que comete mais a raça negra que tem mais incidência desse tipo de anemia. A pessoa tem dores nas articulações porque as hemácias no caso da anemia falciforme é em forma de foice então o organismo identifica como anormal, destrói as hemácias e ai causa a anemia falciforme. Como sabemos, é um tipo de anemia em que não há baixa dos glóbulos vermelhos, mas o glóbulo vermelho ele tem um defeito e o sangue ao passar pelas articulações ele acaba encontrando um pouco mais de resistência onde as pessoas sentem em geral muitas dores articulares. No rastreamento da Triagem Neonatal básica, se der alterações vai ser feito outro exame completo das outras doenças. (E2, E3, E6, E7, E8, E9, E11). 


\section{DISCUSSÃO}

A enfermeira desempenha importante função na implementação do PNTN, sobretudo nas equipes de ESF, pois tem uma maior aproximação com as mães, acompanhando as gestantes desde o pré-natal até o final do período puerperal ${ }^{(4,9)}$. Além disso, a enfermeira possui como atribuições primordiais as ações de promoção, prevenção e manutenção da saúde.

Na prática educativo-assistencial sobre o exame de $\mathrm{TN}$, a enfermeira necessita disponibilizar informações corretas e completas, pois ao compreenderem a importância do exame e as consequências de não diagnosticar precocemente as doenças triadas, provavelmente, os pais e/ou familiares, atuarão também como promotores do crescimento e desenvolvimento saudável de seus filhos ${ }^{(5)}$.

No presente estudo, ao discursarem sobre o que seria o exame de triagem neonatal, as enfermeiras assinalaram pontos importantes que compõem o exame, como o rastreamento, o diagnóstico e/ou descoberta de agravos que podem acometer o desenvolvimento dos recém-nascidos, mas não mencionaram as próximas etapas que se seguem à coleta no PNTN como, por exemplo, a importância do envio da amostra ao SRTN do estado, o repasse dos resultados aos pais ou familiares da criança e a busca ativa dos casos suspeitos ${ }^{(3)}$.

Apesar de já ter se passado mais de uma década da implantação do PNTN no Brasil há estudos que corroboram a existência de algumas fragilidades quanto ao conhecimento da equipe de enfermagem sobre o Teste do Pezinho( ${ }^{(9,11-12)}$.

Um dos exemplos é um estudo realizado em uma Unidade de Tratamento Intensivo Neonatal em um município no estado de São Paulo, o qual objetivou identificar a percepção da equipe de enfermagem acerca da TN. Neste estudo, os autores apontaram que os entrevistados apensar de dominarem termos como identificar, detectar, ver, diagnosticar, tiveram dificuldade em citar a real importância da $\mathrm{TN}^{(11)}$. Segundo concluiu outro estudo ${ }^{(12)}$, esta dificuldade se deve ao fato de que grande parte das orientações sobre o Teste do Pezinho são dadas apenas pelo profissional médico e durante a internação hospitalar.

Destarte, o êxito do PNTN depende da atuação multiprofissional e de forma transversal de todos os profissionais de saúde, principalmente no período pré-natal, no qual as futuras mães e familiares estão mais dispostos a interagir e trocar informações relevantes quanto a medidas preventivas e de qualidade de vida ${ }^{(13)}$.
Ainda, para obter os resultados desejados, o sistema de coleta das amostras requer cuidados especiais sendo importantes, direta ou indiretamente, todas as atividades desenvolvidas, desde a escolha e treinamento do profissional que fará a coleta até a organização do sistema de transporte das amostras para o laboratório que vai realizar as análises ${ }^{(1-3)}$.

A coleta da amostra sanguínea é a primeira de uma série de etapas que fazem parte do processo preventivo $^{(3)}$, sendo recomendável que a captação da mesma se dê após 48 horas da ingestão proteica do recém-nascido, tanto por meio do aleitamento materno como por nutrição parenteral ${ }^{(2-4)}$. Ademais, o PNTN recomenda que a coleta ocorra, preferencialmente, na primeira semana de vida do bebê $\hat{e}^{(1)}$.

Todavia, na presente pesquisa, observou-se que as enfermeiras entrevistadas mencionaram saber o período propício para realizar a coleta, mas não demonstraram segurança sobre a relevância de ser realizada dentro desta janela. Pois, respeitar o período neonatal para realizar a coleta da TN é importante uma vez que a criança pode nascer com alguma das patologias triadas, como o hipotireoidismo congênito, que pode se manifestar a partir da quarta semana de vida do bebê se o tratamento não for precocemente iniciado ${ }^{(3)}$. Assim sendo, torna-se evidente a importância da equipe de enfermagem incluir nas orientações aos pais, não só informações quanto à realização do exame, mas também quanto ao período adequado para que o mesmo seja efetuado ${ }^{(1-4)}$.

Tal qual mencionado pelas entrevistadas no presente estudo, quando a criança permanecer hospitalizada por algum tempo após o nascimento, poderá ser a coleta protelada até que ela esteja em melhores condições para que a mesma ocorra. Porém, esse período não deve ultrapassar os 30 dias de vida da criança ${ }^{(3)}$. Nos casos de prematuridade, a amostra deverá ser coletada da forma habitual nos primeiros dias de vida, sendo feita outra coleta, após 90 dias, para a confirmação do resultado ${ }^{(3-4)}$.

Apesar da literatura estipular um prazo de 30 dias depois do nascimento para a realização do exame, ao contrário do que afirmaram as entrevistadas, há restrições quanto a efetuar a coleta após este período, pois, se por ventura, for diagnosticada alguma das doenças rastreadas com o exame, a criança poderá ficar prejudicada, devido ao retardo da confirmação diagnóstica e início do tratamento ${ }^{(3,12)}$.

Além do período ideal, outra questão destacada pelas enfermeiras entrevistadas, refere-se à técnica 
de coleta. Segundo o Ministério da Saúde ${ }^{(1)}$, a técnica correta de coleta das amostras de sangue para o exame de TN é um procedimento de enfermagem e em todo o local de realização do Teste Pezinho precisa haver, pelo menos, um técnico de enfermagem e uma enfermeira, devidamente capacitados. Além disso, a enfermeira necessita saber orientar e fazer a coleta, pois é imprescindível seguir criteriosamente a técnica adequada ${ }^{(11-13)}$.

Conforme o Manual de Normas Técnicas e Rotinas Operacionais do PNTN $^{(1)}$, para obter um bom resultado na coleta da amostra, cabe ao profissional que fará a mesma: - se necessário, aquecer o pezinho do bebê com bolsa de água morna ou compressa morna, para melhor obtenção do sangue; - fazer anti-sepsia no local correto do pé esquerdo com o algodão ligeiramente umedecido em álcool 70\%; - puncionar com lanceta estéril e descartável num movimento único e firme; - desprezar sempre a primeira formação de gota de sangue; - não comprimir demasiadamente o local de coleta evitando a hemólise; - iniciar a coleta deixando sempre o sangue pingar no papel filtro, observando os círculos e o preenchimento completo nos dois lados do papel.

No entanto, observou-se nos depoimentos que, apesar das enfermeiras terem mencionado o recebimento de treinamentos e tido acesso aos protocolos que orientam a forma correta de realizar o Teste do Pezinho, foram descritos alguns procedimentos equivocados que podem invalidar as amostras tornando necessário reconvocar a criança para nova coleta, ocasionando o retardo no início do tratamento e trazendo prejuízo à mesma.

Perante tais depoimentos, verificou-se a necessidade dos gestores dos serviços de saúde estimularem as enfermeiras envolvidas na coleta do exame de TN para que as mesmas realizem capacitações via educação permanente em saúde e se comprometam com a manutenção da qualidade das ações de enfermagem. Não se pode deixar de considerar que o tempo prolongado de atuação numa mesma função, ou de formação, pode favorecer o desenvolvimento de procedimentos de maneira rotinizada ${ }^{(2)}$ e isso pode ser prejudicial caso não se invista em qualificação permanente.

Do mesmo modo, em locais de maior rotatividade de enfermeiras, o processo de captação das amostras necessita ser constantemente estudado para não comprometer a efetividade do exame. O estímulo para a busca de atualização e qualificação profissional pode se refletir na prática dos enfermeiros, uma vez que, o profissional consciente da importância e finalidade de suas ações, tende a valorizar cada ato durante a prestação do cuidado(4).
Quanto às patologias diagnosticadas a partir da amostra sanguínea coletada pela enfermagem todas as depoentes souberam citar pelo menos as três doenças que tem cobertura pelo PNTN no SRTN do Estado do Rio grande do Sul. Da mesma maneira, as entrevistadas discorreram sobre cada uma das três patologias, assinalando alguns aspectos sobre seu percurso agravante, caso não sejam diagnosticadas e tratadas precocemente.

A plena ciência da finalidade do exame é fundamental para o sucesso do programa, pois, traz mais eficiência ao processo atingindo com qualidade a totalidade dos recém-nascidos ${ }^{(12)}$. Contudo, não basta o saber teórico sobre os procedimentos e as patologias envolvidas se este conhecimento não for compartilhado com pacientes e familiares. Com efeito, um estudo realizado com puérperas ${ }^{(9)}$ observou que $89 \%$ delas demonstraram conhecer o Teste do Pezinho, porém, não sabiam citar quais eram as doenças que o exame identifica e os possíveis agravos ao recém-nascido, caso não fosse feito o exame e detectadas precocemente as enfermidades.

Assim, a partir do relato das entrevistadas e de estudos que reforçaram a fragilidade das informações e do comprometimento profissional das profissionais que interagem com a população alvo do $\operatorname{PNTN}^{(5-6,9)}$, é possível afirmar que o grau de ciência sobre a finalidade do exame, por parte das mães e familiares, resulta da forma como as orientações são passadas pelos profissionais da saúde, no caso, pela enfermeira, pois, para existir prevenção, é indispensável que as pessoas tenham uma adequada informação.

\section{CONCLUSÃO}

Os discursos obtidos evidenciaram o conhecimento das enfermeiras entrevistadas sobre a $\mathrm{TN}$, bem como sua finalidade, período ideal para coleta de sangue, a técnica de coleta, assim como quanto às doenças que são rastreadas e quais são as que o Estado do RS está habilitado para diagnosticar. No entanto, tal conhecimento revelou-se frágil, pois foi tratado pelas entrevistadas muito sucintamente e com pouca sustentação teórica quando respondidas as indagações.

Assim, acredita-se que o enfermeiro, líder de equipe, necessita refletir sobre seu papel social e buscar, continuamente por capacitação. Por sua vez, os gestores públicos precisam se mostrar estimulados a proporcionar maiores oportunidades de qualificação por meio da educação permanente dos profissionais 
de saúde nas diferentes esferas de atenção à saúde no Brasil, a fim de fortalecer o acesso da população à TN, conforme preconiza o PNTN.

Saliente-se que, pelo surgimento constante de inovações tecnológicas na área de diagnóstico de doenças congênitas, os profissionais de saúde devem estar devidamente capacitados e atualizados, para uma adequada orientação aos pais e familiares no que concerne a $\mathrm{TN}$ e às patologias correlatas, com suas possíveis evoluções e tratamentos.

Assim, o presente estudo vem contribuir com a ciência da enfermagem, pois aborda aspectos importantes para a melhoria da prática assistencial, educativa e gerencial do enfermeiro. Este conhecimento pode influenciar positivamente na implementação dos programas de $\mathrm{TN}$.

Contudo, a presente pesquisa possui limites, pois foi realizada somente com enfermeiras que atuam na atenção primária. Para uma maior compreensão do problema proposto, sugere-se realizar outros estudos, abordando diferentes cenários e incluindo os demais profissionais de saúde que também participem da realização da TN.

\section{REFERÊNCIAS}

1. Sociedade Brasileira de Triagem Neonatal. Triagem: passo a passo. [Internet] [acesso em 10 jun 2013]. Disponível: http://www.sbtn.org.br/pg_triag_ doencastratam.htm

2. Ministério da Saúde (BR). Programa Nacional de Triagem Neonatal: Metas para 2013. [Internet] [Acesso em 15 jan 2013]. Disponível: http://portal.saude.gov.br/ portal/arquivos/pdf/2h_280213.pdf

3. Ministério da Saúde (BR). Programa Nacional de Triagem Neonatal. [Internet] [acesso em 15 mai 2013]. Disponível: http://portal.saude.gov.br/portal/saude/area. cfm?id area $=106$

4. Núcleo de Ações e Pesquisa em Apoio Diagnóstico. Diagnóstico Situacional do Programa Nacional de Triagem Neonatal nos estados brasileiros: Belo Horizonte; 2013. 34 p. Relatório técnico.

5. Acosta DF, Strefling ISS, Gomes VLO. Triagem neonatal: (re)pensando a prática de enfermagem. Rev enferm UFPE online. [Internet] 2013;7(2) [acesso em 28 mar 2013]. Disponível: http://www.revista.ufpe. $\mathrm{br} / \mathrm{revistaenfermagem/index.php/revista/article/view}$ Article/3579

6. Abreu IS, Braguini WL. Triagem neonatal: o conhecimento materno em uma maternidade no interior do Paraná, Brasil. Rev. Gaúcha Enferm. [Internet] 2011;32(3) [acesso em 20 jul 2012]. Disponível: http:// dx.doi.org/10.1590/S1983-14472011000300023

7. Leão L, Aguiar M. Newborn screening: what pediatricians should know. J Pediatr. [Internet] 2008;84(4) [acesso em 15 mai 2012]. Disponível: http:// dx.doi.org/10.1590/S0021-75572008000500012

8. Salles M, Santos IMM. O conhecimento das mães acerca do teste do pezinho em uma unidade básica de saúde. Rev. Pesqui Cuid Fundam. [Internet] 2009;1(1) [acesso em 10 nov 2012] Disponível: http://www.seer. unirio.br/index.php/cuidadofundamental/article/view/ $281 / 273$

9. Santos EC, Gaíva MAM, Santos JG, Abud SM. O conhecimento de puérperas sobre a triagem neonatal. Cogitare enferm. [Internet] 2011;16(2) [acesso em 27 set 2012]. Disponível: http://ojs.c3sl.ufpr.br/ojs-2.2.4/ index.php/cogitare/article/viewfile/21817/14227

10. Lefrève $F$, Lefrève AMC. O Discurso do sujeito coletivo: um novo enfoque em pesquisa qualitativa (desdobramentos). $2^{\mathrm{a}}$ ed. Caxias do Sul: Educs; 2005.

11. Benincasa TO, Oliveira CB, Zanoni IH, Lima SAO, Martins DC. Triagem Neonatal: a percepção teórica da equipe de enfermagem da Unidade de Terapia Intensiva Neonatal. Rev Inst Ciênc Saúde. [Internet] 2009;27(2) [acesso em 30 nov 2012]. Disponível: http://files.bvs.br/ upload/S/0104-1894/2009/v27n2/a002.pdf

12. Botler J, Camacho LAB, Cruz MM, George P. Triagem neonatal - o desafio de uma cobertura universal e efetiva. Ciênc. saúde colet. [Internet] 2010;15(2) [acesso em 20 nov 2012]. Disponível: http://dx.doi.org/10.1590/ S1413-81232010000200026

13. Silva CS. O conhecimento das mães e do enfermeiro acerca da Triagem Neonatal. [monografia]. Porto Alegre (RS): Universidade Federal do Rio grande do Sul-UFRGS; 2008. 